\title{
Systemic and mucosal mobilization of granulocyte subsets during lentivirus infection
}

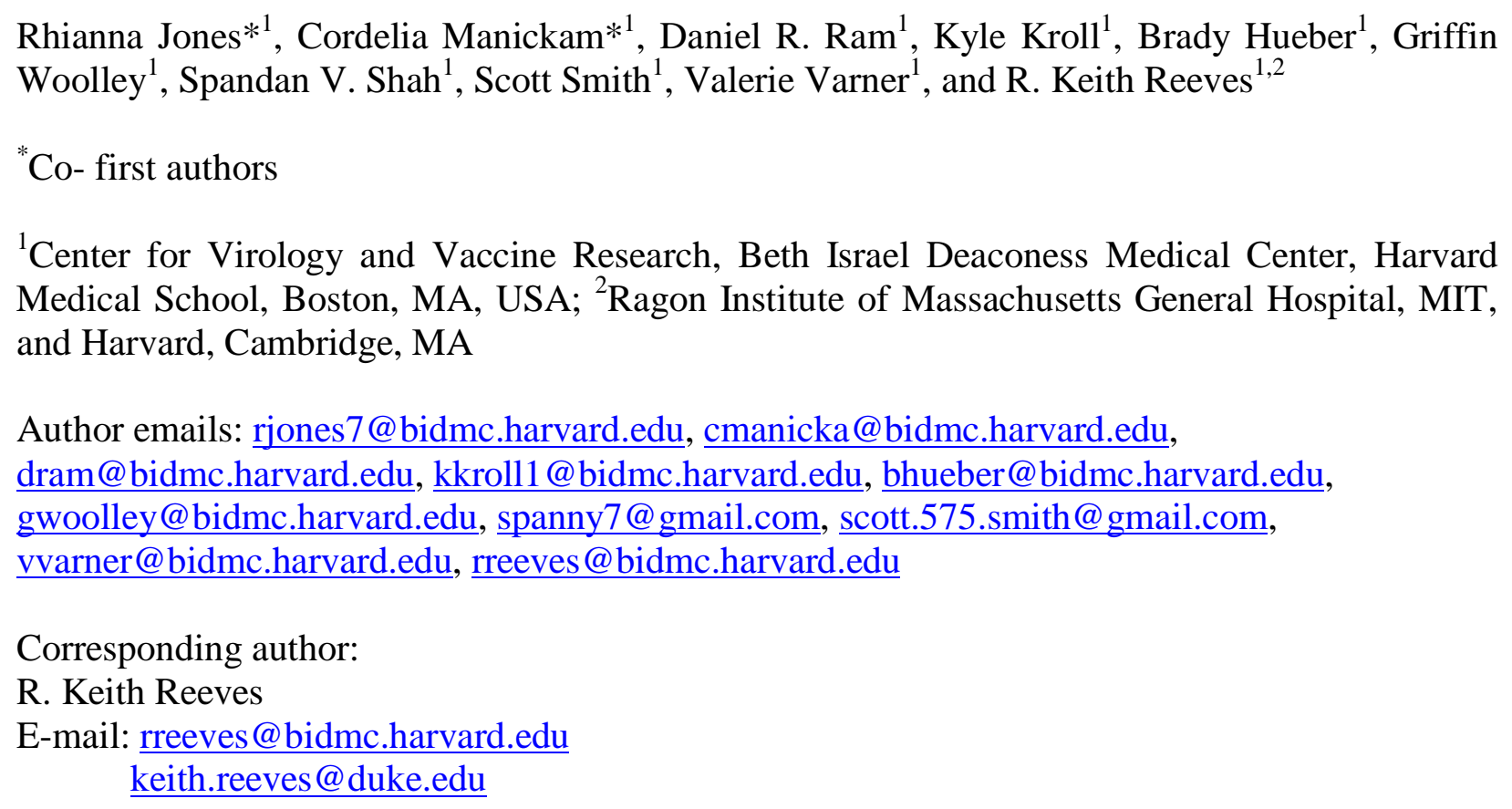

Rhianna Jones* ${ }^{1}$, Cordelia Manickam* ${ }^{1}$, Daniel R. Ram ${ }^{1}$, Kyle Kroll ${ }^{1}$, Brady Hueber ${ }^{1}$, Griffin Woolley ${ }^{1}$, Spandan V. Shah ${ }^{1}$, Scott Smith ${ }^{1}$, Valerie Varner ${ }^{1}$, and R. Keith Reeves ${ }^{1,2}$

${ }^{*}$ Co- first authors

${ }^{1}$ Center for Virology and Vaccine Research, Beth Israel Deaconess Medical Center, Harvard Medical School, Boston, MA, USA; ${ }^{2}$ Ragon Institute of Massachusetts General Hospital, MIT, and Harvard, Cambridge, MA

Author emails: rjones7@ bidmc.harvard.edu, cmanicka@bidmc.harvard.edu, dram@bidmc.harvard.edu, kkroll1@bidmc.harvard.edu, bhueber@bidmc.harvard.edu, gwoolley@bidmc.harvard.edu, spanny7@gmail.com, scott.575.smith@ gmail.com, vvarner@bidmc.harvard.edu, rreeves@bidmc.harvard.edu

Corresponding author:

R. Keith Reeves

E-mail: rreeves@bidmc.harvard.edu

keith.reeves@duke.edu

Running Title: Granulocytes in SHIV-infected macaques

Abstract: X words

28 Text: $\mathrm{X}$ words

29 Figures: 5

30 Tables: 0

Funding statement: The efforts of the authors were supported by NIH grants R01 DE026327, P01 AI120756, R21 AI145678 (all to R.K.R.), as well as the Harvard University Center for AIDS Research Advanced Laboratory Technologies Core (P30 AI060354). 


\section{Abstract}

2 Granulocytes mediate broad immunoprotection through phagocytosis, extracellular

3 traps, release of cytotoxic granules, antibody effector functions and recruitment of other

4 immune cells against pathogens. However, descriptions of granulocytes in HIV infection

5 and mucosal tissues are limited. Our goal was to characterize granulocyte subsets in

6 systemic, mucosal and lymphoid tissues during lentivirus infection using the rhesus

7 macaque (RM) model. Mononuclear cells from jejunum, colon, cervix, vagina, lymph

8 nodes, spleen, liver, and whole blood from naïve, and chronically SHIVsf162p3-infected

9 RM were analyzed by microscopy and polychromatic flow cytometry. Granulocytes were

10 identified using phenotypes designed specifically for RM: eosinophils -

11 CD45+CD66+CD49d+; neutrophils - CD45+CD66+CD14+; and basophils -

$12 \mathrm{CD} 45+\mathrm{CD} 123+\mathrm{FcR} \varepsilon+$. Nuclear visualization with DAPI staining and surface marker

13 images by ImageStream (cytometry/microscopy) further confirmed granulocytic

14 phenotypes. Flow cytometric data showed that all RM granulocytes expressed CD32

15 (FcRyll) but did not express CD16 (FcRyllI). Additionally, constitutive expression of

16 CD64 (FcRyl) on neutrophils and FcRe on basophils, indicates the differential

17 expression of Fc receptors on granulocyte subsets. Granulocytic subsets in naïve whole

18 blood ranged 25.4-81.5\% neutrophils, $0.59-13.3 \%$ eosinophils and $0.059-1.8 \%$

19 basophils. Interestingly, elevated frequencies of circulating neutrophils, colorectal

20 neutrophils, and colorectal eosinophils were all observed in chronic lentivirus disease.

21 Conversely, circulating basophils, jejunal eosinophils, vaginal neutrophils, and vaginal

22 eosinophils of SHIVsf162p3-infected RM declined in frequency. Overall, our data

23 suggest modulation of granulocytes in chronic lentivirus infection, most notably in the 
1 gastrointestinal mucosae where significant inflammation and disruption occurs in

2 lentivirus-induced disease. Furthermore, granulocytes may migrate to inflamed tissues

3 during infection and could serve as targets of immunotherapeutic intervention. 


\section{Introduction:}

2 Granulocytes, which include neutrophils, eosinophils, and basophils are critical innate

3 immune cells that form one of the first lines of immune defense against pathogens.

4 They predominantly act against bacterial $(4,11,19,33,38,39)$, and parasitic pathogens

$5(119,24,37,39)$, and although granulocytes are also active against viral pathogens (2,

$65,7,11,14,16,31,33,37,38)$, this is less well characterized. Among the subsets,

7 neutrophils are generally the most abundant and well-characterized $(4,14,33,47)$,

8 capable of multiple specialized methods of immune defense. In response to pathogen-

9 induced inflammation $(4,37,39,47)$, neutrophils release cytokines, neutrophil

10 extracellular traps (NETs), and activate phagocytosis $(2,19,38,39,47)$. In response to

11 viral pathogens, such as HIV, $(11,33,37,38)$, neutrophils activate NETs in the female

12 genital tract and other tissues, perform phagocytosis and release reactive oxygen

13 species $(2,14,32,38)$. In some cases, overly robust neutrophil responses can also

14 cause tissue damage and further disease pathogenesis as evidenced in persons living

15 with HIV (PLWH) and SIV-infected nonhuman primates (NHPs) $(5,14,32,35,45)$.

16 Conversely, granulocyte frequencies and functions may be abrogated by HIV disease

$17(5,13,31)$, including neutrophil dysregulation and dysfunction (31) and a decline in

18 frequency due to HIV accelerated apoptosis and hematopoietic failure in the bone

19 marrow $(5,13,31)$. Similar accelerated neutrophil apoptosis mediated by viral infection

20 has also been observed in rhesus macaques (RM) infected with SIV $(7,8,16)$.

21 Eosinophils and basophils can also respond to pathogens through the release of

22 granules and cytokines, and in some cases through extracellular traps $(19,24,26,38$,

23 39). While both eosinophils and basophils are primarily associated with allergens and 
1 parasitic infections, they may be effective antigen presenting cells (APCs), similar to

2 neutrophils, and help in activating Th2 responses $(21,23,26,47)$. Additionally,

3 eosinophils and basophils can interact with dendritic cells and B cells directly and/or

4 indirectly through $\mathrm{T}$ cell activation to promote Th2 immunity, B cell proliferation and

5 antibody production $(26,37)$. There has also been evidence that eosinophils play a role

6 in limiting inflammation, tissue glucose uptake and minimizing host tissue damage (1,

7 37). However, similar to the effect on neutrophils, HIV may cause an expansion of

8 eosinophils associated with a decrease in CD4+ T cells and in parasitic coinfections 9 (31).

10 NHPs have long been used as models of immunological research because of their

11 similarities to human physiology, immunology and disease pathogenesis $(3,12,16,20$,

12 32). Granulocyte immune function similarities between human and NHP models include

13 immune cell recruitment $(12,14,24,32)$, phagocytosis $(16,17,32,36,39)$, and cytokine

14 and chemokine release $(10,23,32)$. However, phenotypic differences between human

15 and macaque granulocytes must also be considered. In humans, CD16 is the preferred

16 cell marker for identifying neutrophils, but NHP neutrophils do not express CD16. Thus,

17 CD14 has previously been used to partially identify NHP neutrophils $(16,32,47,48)$.

18 Healthy rhesus macaques can have a population of neutrophils ranging from $20-50 \%$ of

19 all white blood cells (WBC) (12), whereas in humans this range is $40-70 \%$ neutrophils of

20 all WBC $(16,17,33)$. The frequencies of basophils $(<1 \%$ of WBC) and eosinophils $(0.3-$

$2110 \%$ of WBC) populations are generally similar in humans and macaques $(12,31)$.

22 Regardless, consensus phenotypes for each population in NHPs, particularly in tissues,

23 are lacking. Therefore, in this study, we performed in-depth phenotypic analysis of 
1 neutrophils, eosinophils, and basophils in systemic and mucosal tissues of naïve and

2 SHIV-infected RM using flow cytometry and ImageStream cytometry.

4 Methods

5 Animal samples:

6 Indian-origin rhesus macaques (Macaca mulatta) were used for all components of this

7 study and included: experimentally naïve $(n=19)$, or chronically infected with

8 SHIVsf162p3 $(n=21)$. Blood, biopsies and other tissues were collected where

9 indicated and processed using standard protocols. All animals were housed at either

10 Biomere, Inc. (Worcester, MA) or Bioqual, Inc. (Rockville, MD). All study collections

11 were reviewed and approved by either the Biomere Institutional Animal Care and Use

12 Committee or the Bioqual Institutional Animal Care and Use Committee.

\section{Tissue processing and preparation:}

15 Mucosal biopsies (colorectal and vaginal) and tissues (vagina, cervix, colon, jejunum,

16 colorectal) were enzymatically digested with collagenase (type IV for reproductive

17 tissues, type II for digestive tissues), pushed through $70 \mu \mathrm{m}$ nylon mesh cell filters and

18 then were overlaid on $35 \%$ percoll (Sigma-Aldrich) followed by $60 \%$ percoll for density

19 gradient centrifugation. Liver tissue was mechanically disrupted and pushed through cell

20 filters and was similarly subjected to density gradient centrifugation. The interphase

21 containing leukocytes was collected, washed with RPMI 1640 (Corning) containing 5\%

22 fetal bovine serum (FBS), counted and used for phenotyping granulocytes. Lymph

23 nodes and spleen were mechanically pushed through $70 \mu \mathrm{m}$ nylon mesh cell filters 
1 followed by RBC lysis with ACK (ammonium-chloride-potassium) lysing buffer

2 (ThermoFisher Scientific) solution. The cells were washed and resuspended in RPMI

31640 containing $10 \%$ FBS for further use in assays.

5 Flow cytometric staining and analysis of whole blood and tissue samples:

6 To identify granulocytes using polychromatic flow cytometric staining, $100 \mu \mathrm{L}$ of whole

7 blood was stained with surface antibodies for 20 minutes at room temperature,

8 protected from light. Antibodies against the following molecules were included: CD49d

9 (HP2/1; Beckman Coulter), CD32 (FUN-2; BioLegend), CD95 (DX2; BD Pharmingen),

10 CD117 (104D2; BioLegend), CD123 (6H6; BioLegend), CD20 (2H7; BioLegend),

11 CD11b (ICRF44; BD Pharmingen), CD14 (M5E2 BD; Pharmingen), CD62L (SK11; BD

12 Pharmingen), CD45 (D058-1283; BD Pharmingen), CD3 (SP34.2; BD Pharmingen),

13 CD16 (3G8; BD Pharmingen), CD64 (10.1; BD Pharmingen), CD66abce (TET2;

14 Miltenyi), CD63 (H5C6; BioLegend), HLA-DR (G46-6; BD Pharmingen), and FcRe

15 (AER-37 (CRA-1); BioLegend). Following this, FACS lysis buffer (BD Biosciences) was

16 used to lyse red blood cells (RBC) according to manufacturer's recommendations.

17 Samples were then washed twice with wash buffer (1X DPBS containing 2\% FBS) and

18 fixed with $1 \%$ formaldehyde. Cells from processed tissues were first stained with

19 LIVE/DEAD fixable aqua stain (Life Technologies) to distinguish live cells. After 20

20 minutes of incubation at $4{ }^{\circ} \mathrm{C}$, cells were washed and stained for surface markers as

21 listed above and incubated at $4 \square \mathrm{C}$ for 20 minutes. Finally, cells were fixed using $1 \%$

22 formaldehyde. Data were acquired using an LSRII flow cytometer (BD Biosciences) and

23 analyzed using FlowJo software (version 10.6.1). 


\section{ImageStream flow cytometric staining and analysis:}

3 Whole blood and tissue mononuclear cells were stained for ImageStream cytometry

4 similar to above with the same antibodies. Two panels were used to characterize

5 neutrophils, eosinophils, and basophils. Markers used for the eosinophil/neutrophil

6 panel were: CD49d, CD45, CD14, CD66abce, and HLA-DR and markers used for

7 basophil panel were CD45, CD66abce CD123, HLA-DR, and FcRe. After 30 minutes of

8 incubation with surface antibodies, cells were washed and stained with DAPI

9 (Thermofisher) to visualize nuclear morphology after permeabilization with Invitrogen

10 Fix and Perm medium kit (Thermofisher) for whole blood staining. In case of tissue

11 immune cells, DAPI was used for live and dead cell discrimination without

12 permeabilization. After fixation with $1 \%$ formaldehyde, samples were recorded using an

13 ImageStreamX Mk II (EMD Millipore) and analyzed using IDEAS Application v6. The

14 general analysis, colocalization and internalization modules in the IDEAS software were

15 utilized in analyzing these samples.

16

\section{Statistical Analyses:}

18 Comparisons between naïve and SHIV-infected animals were analyzed by Mann-

19 Whitney $U$ tests using Prism v.8 software. Differences between test groups were

20 considered significant when $p<0.05$.

\section{Results:}

23 Myeloid cell phenotypes in whole blood identified using flow cytometry. 
1 Initial identification of granulocyte populations (and monocytes for comparison) was

2 determined among medium to high side scatter (granularity) CD45+ leukocytes.

3 Subsequently cells were divided into those that express the CD66 family of receptors

4 (eosinophils, neutrophils) and those that do not (basophils). Next, based on previous

5 reports $(16,32,47,48)$, CD14 was used as a general marker to distinguish neutrophil

6 populations from other subsets and each granulocyte population was defined as follows:

7 CD45+HLA-DR-CD14+CD49d-CD66+ neutrophils, CD45+HLA-DR-CD14-

8 CD49d+CD66+ eosinophils, and CD45+HLA-DR-CD123+CD66- basophils (Fig. 1a).

9 Monocytes were defined as HLA-DR+CD14+CD16+/- to provide discrimination of all

10 myeloid cell phenotypes in whole blood of RM (Fig. 1 and 1b). To further confirm

11 phenotypic identity in $\mathrm{RM}, \mathrm{Fc}$ receptor expression was compared among all populations.

12 As expected, all subsets had high frequencies of CD32 (FcRyll; Fig.1b). CD64 (FcRyl)

13 was expressed at variable levels on all populations except basophils, but as expected,

14 basophils were the only cells to demonstrate high constitutive expression of FcRe. In

15 strong agreement with previous studies for RM (18), CD16 (FcRy III) was not

16 expressed on granulocytes but only on a subset of monocytes. Cell frequencies in

17 whole blood of experimentally naïve RM were analyzed to evaluate baseline

18 frequencies (Fig. 1c), and as expected, neutrophils had the highest frequency out of the

19 three subsets (median 51.1, range 25.4-81.5\%), followed by eosinophils (median 3.16,

20 range $0.59-13.3 \%$ ) and basophils (median 0.048 , range $0.059-1.8 \%$ ).

22 Granulocyte morphology and surface phenotypes by ImageStream cytometry. 
1 To further confirm the identity of RM granulocyte subpopulations, we next evaluated

2 bulk and individual cells phenotypically and morphologically by ImageStream cytometry.

3 Whole blood samples were stained for DAPI to identify nuclear morphology and surface

4 markers to confirm surface expression of markers used in the standard flow cytometry

5 panels. Using the general analysis wizard of the IDEAs Application v6, DAPI+ cells were

6 selected and representative analyses are shown in Figure 2. As in Figure 1, neutrophils

7 were confirmed by surface co-expression of CD14 and CD66, eosinophils by co-

8 expression of CD49d and CD66, and basophils by co-expression of $\mathrm{FcR} \varepsilon$ and CD123

9 (Fig. 3). Further analysis of internalization in the IDEAs software confirmed the nuclear

10 morphology of the granulocyte populations. Analysis of DAPI+ cells showed multilobed

11 nuclei of CD14+CD49d-CD66+ neutrophil populations, bilobed nuclei of CD14-

12 CD49d+CD66+ eosinophils and granular cytoplasm of basophils as expected (Fig. 3).

14 Modulation of peripheral and tissue granulocytic subsets in infected animals.

15 In a cross-sectional evaluation of naïve RM and chronically SHIV-infected RM, we first

16 quantified granulocytes in whole blood. Circulating neutrophils were increased over

$1750 \%$ in SHIV-infected animals compared to naïve RM (medians, $79.6 \%$ versus $51.1 \%$ ).

18 While circulating eosinophils were largely unaltered, basophils were modestly, but

19 significantly $(p=0.003)$ reduced in infected animals (Fig 4).

20 Next we applied our analyses of granulocytes in whole blood to tissues collected by

21 mucosal biopsy or at necropsy from both naïve and chronically SHIV-infected animals

22 and observed neutrophils and eosinophils in vaginal biopsies of naïve animals

23 (medians, $0.65 \%$ and $0.38 \%$ respectively) (Fig. 5a). Interestingly, in SHIV-infected RM, 
1 both cell types were significantly reduced. Neutrophils and eosinophils were both found

2 at low frequencies in colorectal biopsies of naive animals (medians, $0.083 \%$ and

$30.021 \%$ respectively), but were significantly increased during infection (both 3-fold

4 increases).

5 Quantification of granulocytes in a very small subset of the animals in spleen, liver,

6 colon, jejunum, and axillary lymph nodes from necropsy tissues revealed detectable

7 populations in most animals (Fig. 5b). Following infection we were unable to detect

8 significant changes, most likely due to the low number of animal samples. In particular,

9 basophil frequencies were near the threshold of detection in tissues, and aside from one

10 animal these frequencies were also unchanged during infection. Notably, both

11 eosinophils and neutrophils seem to increase in liver tissue, but larger studies will be

12 needed to validate this finding. We also noted an interestingly high frequency of

13 eosinophils in jejunum of naive animals (median, 47\%) which could also be of interest in

14 future studies.

\section{Discussion:}

17 Granulocytes play major roles as innate immune cells against bacterial, viral and

18 parasitic pathogens which have been described in multiple studies $(11,19,24,33,38$,

19 39). Their protective role against HIV as well as their pathogenic role causing tissue

20 damage in HIV infected patients, particularly by neutrophils, have been reported in

21 multiple studies $(12,16,17,31,33,47,48)$. However, NHP granulocytes in mucosal

22 tissues of chronic SHIV infections have been underexplored. Here, we describe

23 granulocyte subset modulations in systemic and mucosal tissues of SHIV infected RM. 
1 Using flow cytometry, we first identified the phenotype of granulocytes as

2 CD14+CD49d-CD66+ neutrophils, CD14-CD49d+CD66+ eosinophils, and

3 CD123+CD66- basophils. A major difference in phenotype between human and NHP

4 neutrophils is the expression of CD14 by NHP neutrophils rather than CD16 as seen in

5 human neutrophils. Indeed, a subset of monocytes were the only myeloid cells to

6 express CD16 in NHP. Due to the limited availability of cross-reactive antibodies to

7 further characterize granulocytic subsets, we confirmed the granulocyte phenotype by

8 examination of their nuclear morphology and surface marker expression. ImageStream,

9 a tool that combines microscopy and flow cytometry, allowed us to visualize the

10 polymorphonuclear neutrophils, bilobed eosinophil nuclei and granular basophils (Fig. 2

11 and 3), thus confirming the granulocyte phenotypes we have identified for rhesus

12 macaques.

13 Persistent immune activation is commonly associated with chronic HIV infection.

14 Studies examining neutrophils in both acute and chronic SIV infection $(13,32)$ showed

15 altered functional and phenotypic differences in CD11b+ neutrophils, however a

16 reduction of these cells were seen in acute infection. Similarly, in people living with HIV,

17 neutropenia $(22,29,30,41,46)$, and impaired phagocytic activity and cytokine

18 production $(10,27,30,36)$ have been frequently observed. Interestingly, we observed

19 significantly increased neutrophils and decreased basophils compared to naïve animals

20 in the blood of SHIV-infected animals. While basophils are predominantly located in the

21 blood, our observed decline in basophils may be due to their infiltration of inflamed

22 tissues as seen in the gastric mucosa of Helicobater pylori infected patients (6) and in

23 the skin of the cutaneous allergic reactions (40). 
1 Progressive HIV infection can cause immune dysfunction in mucosal tissues of the gut

2 and also the reproductive tract $(14,15,43,44,50)$. In this study we found elevated

3 frequencies of neutrophils and eosinophils in colorectal biopsies and higher eosinophil

4 frequency in jejunum samples of chronic SHIV infected animals compared to naïve

5 animals (Fig. 5) indicating granulocytic infiltration of gut tissues. Similar observations of

6 increased neutrophil infiltration and epithelial barrier dysfunction have been reported in

7 the gut tissues of SIV infected animals and treated and untreated chronic HIV infected

8 patients $(9,45)$. Further, Hensley-McBain et al have showed that HIV induced microbial

9 translocation and dysbiosis characterized by reduced Lactobacillus: Prevotella ratio as

10 the reason for the increased neutrophil recruitment and increased neutrophil lifespan in

11 the colorectal mucosa of HIV infected individuals (15). Neutrophil infiltration in the

12 colonic mucosa is also correlated with disease severity in inflammatory bowel disease,

13 thus further suggesting that elevated neutrophils in the gut are potentially inflammatory

14 and induce additional mucosal damage.

15 Interestingly, chronic SHIV infected vaginal biopsies had significantly lower frequencies

16 of neutrophils and eosinophils than naïve animals in our study. This is in accordance

17 with studies that have shown increased neutrophil apoptosis in whole blood and tissues,

18 and eosinophil apoptosis in whole blood during lentivirus infection in humans $(7,8,16$,

19 45, 49). Similarly in PLWH vaginal inflammation was associated with increased

20 neutrophils (33). In vitro data suggests that neutrophils are capable of entrapping and

21 inactivating HIV through NETS within minutes of viral exposure (2). Based on this, our

22 data suggests differential modulation of granulocyte trafficking, expansion and functions

23 of resident granulocyte subsets in different mucosal tissues by chronic SHIV infection. 
1 However, more research exploring granulocyte functions in mucosal tissues, particularly

2 in chronic SIV/SHIV infection is warranted.

3 In summary, our study provides comprehensive data on granulocyte phenotypes in

4 blood and tissues of RM, which was consistent with other reports $(16,25,32)$.

5 Visualization of granulocytes by ImageStream proved to be an excellent tool to confirm

6 immune cell phenotypes. Further, our data showed that mucosal tissues which are the

7 sites of viral infection show modulated frequencies of granulocyte subsets and thereby

8 could differ in their functional outcomes of either immunoprotection or tissue damage.

9 While we analyzed other systemic and lymphoid tissues including spleen, liver and

10 axillary LN, we did not see any changes. The limitations of our study include a small

11 sample size and availability of only cross-sectional data for chronic SHIV infection.

12 Longitudinal time points in chronic SHIV infected animals would provide a better

13 understanding of the granulocyte subset modulations. Overall, our data provides

14 evidence of immune modulation of granulocytes in gut and vaginal tissue and warrants

15 more research into identifying their roles in protection/ pathology in lentivirus infection.

\section{Acknowledgements:}

18 The efforts of the authors were supported by NIH grants R01 DE026327, P01

19 Al120756, R21 Al145678 (all to R.K.R.), as well as the Harvard University Center for

20 AIDS Research Advanced Laboratory Technologies Core (P30 AI060354). The authors

21 also wish to thank Dr. Dan Barouch for providing some animal samples and Michelle

22 Lifton for assistance with flow cytometry. 


\section{Abbreviations:}

2 RM: Rhesus macaques

3 HIV: Human Immunodeficiency Virus

4 SIV: Simian Immunodeficiency Virus

5 SHIV: Simian-Human Immunodeficiency Virus

6 


\section{References:}

2 1. Abdala-Valencia, Hiam, Mackenzie E. Coden, Sergio E. Chiarella, Elizabeth A. Jacobsen,

3 Bruce S. Bochner, James J. Lee, and Sergejs Berdnikovs. "Shaping Eosinophil Identity in the

4 Tissue Contexts of Development, Homeostasis, and Disease." Journal of Leukocyte Biology

5 104, no. 1 (July 2018): 95-108. https://doi.org/10.1002/JLB.1MR1117-442RR.

6 2. Barr, Fiona D., Christina Ochsenbauer, Charles R. Wira, and Marta Rodriguez-Garcia.

7 "Neutrophil Extracellular Traps Prevent HIV Infection in the Female Genital Tract." Mucosal

8 Immunology 11, no. 5 (2018): 1420-28. https://doi.org/10.1038/s41385-018-0045-0.

9 3. Bontrop, Ronald E., and David I. Watkins. "MHC Polymorphism: AIDS Susceptibility in Non-

10 Human Primates." Trends in Immunology 26, no. 4 (April 2005): 227-33.

11 https://doi.org/10.1016/j.it.2005.02.003.

12 4. Castanheira, Fernanda V. S., and Paul Kubes. "Neutrophils and NETs in Modulating Acute 13 and Chronic Inflammation." Blood 133, no. $20 \quad$ (16 2019): 2178-85.

14 https://doi.org/10.1182/blood-2018-11-844530.

15 5. Casulli, Sarah, and Carole Elbim. "Interactions between Human Immunodeficiency Virus 16 Type 1 and Polymorphonuclear Neutrophils." Journal of Innate Immunity 6, no. 1 (2014): 1317 20. https://doi.org/10.1159/000353588.

18 6. de Paulis, Amato, Nella Prevete, Isabella Fiorentino, Andrew F. Walls, Monica Curto, 19 Angelica Petraroli, Vincenza Castaldo, Paola Ceppa, Roberto Fiocca, and Gianni Marone.

20 "Basophils Infiltrate Human Gastric Mucosa at Sites of Helicobacter Pylori Infection, and 21 Exhibit Chemotaxis in Response to H. Pylori- Derived Peptide Hp(2-20)." The Journal of 22 Immunology 172, no. $12 \quad$ (June $15, \quad 2004$ ): $\quad 7734-43$.

23 https://doi.org/10.4049/jimmunol.172.12.7734.

24 7. Elbim, Carole, Valérie Monceaux, Stéphanie François, Bruno Hurtrel, Marie-Anne Gougerot25 Pocidalo, and Jérome Estaquier. "Increased Neutrophil Apoptosis in Chronically SIV-Infected 
1 8. Elbim, Carole, Valerie Monceaux, Yvonne M. Mueller, Mark G. Lewis, Stephanie François, Ousmane Diop, Khadija Akarid, et al. "Early Divergence in Neutrophil Apoptosis between Pathogenic and Nonpathogenic Simian Immunodeficiency Virus Infections of Nonhuman Primates." Journal of Immunology (Baltimore, Md.: 1950) 181, no. 12 (December 15, 2008): 8613-23. https://doi.org/10.4049/jimmunol.181.12.8613.

9. Epple, H-J, T Schneider, H Troeger, D Kunkel, K Allers, V Moos, M Amasheh, et al. "Impairment of the Intestinal Barrier Is Evident in Untreated but Absent in Suppressively Treated HIV-Infected Patients." Gut 58, no. 2 (February 1, 2009): 220-27. https://doi.org/10.1136/gut.2008.150425.

10. Gasperini, S., R. Zambello, C. Agostini, L. Trentin, C. Tassinari, P. Cadrobbi, G. Semenzato, and M. A. Cassatella. "Impaired Cytokine Production by Neutrophils Isolated from Patients with AIDS." AIDS (London, England) 12, no. 4 (March 5, 1998): 373-79. https://doi.org/10.1097/00002030-199804000-00005.

11. Giraldo, Diana M., Juan C. Hernandez, and Silvio Urcuqui-Inchima. "HIV-1-Derived SingleStranded RNA Acts as Activator of Human Neutrophils." Immunologic Research 64, no. 5-6 (2016): 1185-94. https://doi.org/10.1007/s12026-016-8876-9.

12. He, Ziyuan, Carolina Allers, Chie Sugimoto, Nursarat Ahmed, Hideki Fujioka, Woong-Ki Kim, Elizabeth S. Didier, and Marcelo J. Kuroda. "Rapid Turnover and High Production Rate of Myeloid Cells in Adult Rhesus Macaques with Compensations during Aging." Journal of Immunology (Baltimore, Md.: 1950) 200, no. $12 \quad$ (15 2018): 4059-67. https://doi.org/10.4049/jimmunol.1800207.

13. Hensley-McBain, Tiffany, Alicia R. Berard, Jennifer A. Manuzak, Charlene J. Miller, Alexander S. Zevin, Patricia Polacino, Jillian Gile, et al. "Intestinal Damage Precedes Mucosal Immune Dysfunction in SIV Infection.” Mucosal Immunology 11, no. 5 (September 2018): 1429-40. https://doi.org/10.1038/s41385-018-0032-5. 
1 14. Hensley-McBain, Tiffany, and Nichole R. Klatt. "The Dual Role of Neutrophils in HIV

2 Infection." Current HIVIAIDS Reports 15, no. 1 (February 2018): 1-10.

3 https://doi.org/10.1007/s11904-018-0370-7.

4 15. Hensley-McBain, Tiffany, Michael C. Wu, Jennifer A. Manuzak, Ryan K. Cheu, Andrew

5 Gustin, Connor B. Driscoll, Alexander S. Zevin, et al. "Increased Mucosal Neutrophil Survival

6 Is Associated with Altered Microbiota in HIV Infection." Edited by Daniel C. Douek. PLOS $\begin{array}{llllll}\text { Pathogens } & \text { 15, no. } 4 \text { (April } 4 \text { 11, }\end{array}$

$8 \quad$ https://doi.org/10.1371/journal.ppat.1007672.

9 16. Huot, Nicolas, Philippe Rascle, Thalia Garcia-Tellez, Beatrice Jacquelin, and Michaela

10 Müller-Trutwin. "Innate Immune Cell Responses in Non Pathogenic versus Pathogenic SIV 11 Infections." Current Opinion in Virology $19 \quad$ (2016): 37-44.

12 https://doi.org/10.1016/j.coviro.2016.06.011.

13 17. Karsten, Christina B., Nickita Mehta, Sally A. Shin, Thomas J. Diefenbach, Matthew D. Slein, 14 Wiktor Karpinski, Edward B. Irvine, Thomas Broge, Todd J. Suscovich, and Galit Alter. "A 15 Versatile High-Throughput Assay to Characterize Antibody-Mediated Neutrophil Phagocytosis." Journal of Immunological Methods $471 \quad$ (2019): 46-56.

18 18. Kim, Woong-Ki, Yue Sun, Hien Do, Patrick Autissier, Elkan F. Halpern, Michael Piatak, 19 Jeffrey D. Lifson, Tricia H. Burdo, Michael S. McGrath, and Kenneth Williams. "Monocyte 20 Heterogeneity Underlying Phenotypic Changes in Monocytes According to SIV Disease 21 Stage." Journal of Leukocyte Biology 87, no. 4 (April 2010): 557-67. 22 https://doi.org/10.1189/jlb.0209082.

23 19. Kolaczkowska, Elzbieta, and Paul Kubes. "Neutrophil Recruitment and Function in Health and 24 Inflammation." Nature Reviews Immunology 13, no. 3 (March 2013): 159-75. 25 https://doi.org/10.1038/nri3399. 
1 20. Lafont, B. A., L. Gloeckler, J. L. D’Hautcourt, J. P. Gut, and A. M. Aubertin. "One-Round

2 Determination of Seven Leukocyte Subsets in Rhesus Macaque Blood by Flow Cytometry."

3 Cytometry 41, no. 3 (November 1, 2000): 193-202. https://doi.org/10.1002/1097-

$4 \quad \underline{0320(20001101) 41: 3<193:: \text { aid-cyto6>3.0.co;2-g. }}$

5 21. Leliefeld, Pieter H. C., Leo Koenderman, and Janesh Pillay. "How Neutrophils Shape

6 Adaptive Immune Responses." Frontiers in Immunology 6 (2015): 471.

7 https://doi.org/10.3389/fimmu.2015.00471.

8 22. Levine, Alexandra M., Roksana Karim, Wendy Mack, D. Jay Gravink, Katherine Anastos,

9 Mary Young, Mardge Cohen, et al. "Neutropenia in Human Immunodeficiency Virus Infection:

10 Data from the Women's Interagency HIV Study." Archives of Internal

11 Medicinehttps://doi.org/10.1001/archinte.166.4.405.

12 23. Lin, Ang, and Karin Loré. "Granulocytes: New Members of the Antigen-Presenting Cell

13 Family." Frontiers in Immunology 8 (2017): 1781. https://doi.org/10.3389/fimmu.2017.01781.

14 24. Loktionov, Alexandre. "Eosinophils in the Gastrointestinal Tract and Their Role in the

15 Pathogenesis of Major Colorectal Disorders.” World Journal of Gastroenterology 25, no. 27

16 (July 21, 2019): 3503-26. https://doi.org/10.3748/wjg.v25.i27.3503.

17 25. Mercado, Noe B., Roland Zahn, Frank Wegmann, Carolin Loos, Abishek Chandrashekar, 18 Jingyou Yu, Jinyan Liu, et al. "Single-Shot Ad26 Vaccine Protects against SARS-CoV-2 in 19 Rhesus Macaques." Nature 586, no. 7830 (October 22, 2020): 583-88. 20 https://doi.org/10.1038/s41586-020-2607-z.

21 26. Merluzzi, Sonia, Elena Betto, Alice Amaranta Ceccaroni, Raffaella Magris, Marina Giunta, 22 and Francesca Mion. "Mast Cells, Basophils and B Cell Connection Network." Molecular 23 Immunology 63, no. 1 (January 2015): 94-103. https://doi.org/10.1016/j.molimm.2014.02.016.

24 27. Michailidis, C, G Giannopoulos, V Vigklis, K Armenis, A Tsakris, and P Gargalianos. 25 "Impaired Phagocytosis among Patients Infected by the Human Immunodeficiency Virus: 26 Implication for a Role of Highly Active Anti-Retroviral Therapy." Clinical and Experimental 
Immunology 167, no. 3 (March 2012): 499-504. https://doi.org/10.1111/j.13652249.2011.04526.x.

3 28. Mitsumoto-Kaseida, Fujiko, Masayuki Murata, Kazuya Ura, Koji Takayama, Satoshi Hiramine, Motohiro Shimizu, Kazuhiro Toyoda, Eiichi Ogawa, and Norihiro Furusyo. "The Expression Level of Neutrophil CD64 Is a Useful Marker of Systemic Inflammation Associated with HIV Infection." AIDS Research and Human Retroviruses 33, no. 2 (2017): 147-56. https://doi.org/10.1089/AID.2016.0107.

29. Moore, D. A., T. Benepal, S. Portsmouth, J. Gill, and B. G. Gazzard. "Etiology and Natural History of Neutropenia in Human Immunodeficiency Virus Disease: A Prospective Study." Clinical Infectious Diseases: An Official Publication of the Infectious Diseases Society of America 32, no. 3 (February 1, 2001): 469-75. https://doi.org/10.1086/318495.

30. Moore, D. A., A. Sullivan, P. Hilstead, and B. G. Gazzard. "A Retrospective Study of

15 31. Ms, Espíndola, Soares Ls, Galvão-Lima Lj, Zambuzi Fa, Cacemiro Mc, Brauer Vs, and Frantz 16 Fg. "HIV Infection: Focus on the Innate Immune Cells." Immunologic research. Immunol Res, 17 December 2016. https://doi.org/10.1007/s12026-016-8862-2.

18 32. Musich, Thomas, Mohammad Arif Rahman, Venkatramanan Mohanram, Leia Miller-Novak, 19 Thorsten Demberg, David J. Venzon, Barbara K. Felber, Genoveffa Franchini, George N. 20 Pavlakis, and Marjorie Robert-Guroff. "Neutrophil Vaccination Dynamics and Their Capacity 21 to Mediate B-Cell Help in Rhesus Macaques." Journal of Immunology (Baltimore, Md. $\square$ : 22 1950) 201, no. 8 (October 15, 2018): 2287-2302. https://doi.org/10.4049/jimmunol.1800677.

23 33. Naumenko, Victor, Madison Turk, Craig N. Jenne, and Seok-Joo Kim. "Neutrophils in Viral 24 Infection." Cell and Tissue Research 371, no. 3 (March 2018): 505-16. 25 https://doi.org/10.1007/s00441-017-2763-0. 
1 34. Nkwanyana, Nonhlanhla N, Pamela P Gumbi, Lindi Roberts, Lynette Denny, Willem Hanekom, Andreia Soares, Bruce Allan, et al. "Impact of Human Immunodeficiency Virus 1 Infection and Inflammation on the Composition and Yield of Cervical Mononuclear Cells in the Female Genital Tract." Immunology 128, no. 1 Pt 2 (September 2009): e746-57. https://doi.org/10.1111/j.1365-2567.2009.03077.x.

35. Passmore, Jo-Ann S., Heather B. Jaspan, and Lindi Masson. "Genital Inflammation, Immune Activation and Risk of Sexual HIV Acquisition." Current Opinion in HIV and AIDS 11, no. 2 (March 2016): 156-62. https://doi.org/10.1097/COH.0000000000000232.

36. Pugliese, Agostino, Valerio Vidotto, Tiziana Beltramo, and Donato Torre. "Phagocytic Activity in Human Immunodeficiency Virus Type 1 Infection.” Clinical and Diagnostic Laboratory Immunology 12, no. 8 (August 2005): 889-95. https://doi.org/10.1128/CDLI.12.8.889895.2005.

37. Ravin, Karen A., and Michael Loy. "The Eosinophil in Infection." Clinical Reviews in Allergy \& Immunology 50, no. 2 (April 2016): 214-27. https://doi.org/10.1007/s12016-015-8525-4.

38. Saitoh, Tatsuya, Jun Komano, Yasunori Saitoh, Takuma Misawa, Michihiro Takahama, Tatsuya Kozaki, Takuya Uehata, et al. "Neutrophil Extracellular Traps Mediate a Host Defense Response to Human Immunodeficiency Virus-1." Cell Host \& Microbe 12, no. 1 (July 2012): 109-16. https://doi.org/10.1016/j.chom.2012.05.015.

39. Scapini, Patrizia, and Marco A. Cassatella. "Social Networking of Human Neutrophils within the Immune System." Blood 124, no. 5 (July 31, 2014): 710-19. https://doi.org/10.1182/blood2014-03-453217.

40. Schroeder, John T. "Basophils: Emerging Roles in the Pathogenesis of Allergic Disease." Immunological Reviews 242, no. 1 (2011): 144-60. https://doi.org/10.1111/j.1600065X.2011.01023.x.

41. Sharma, Prashant, Santhanam A. V. Raghavan, Rashmi Saini, and Madhu Dikshit. "Ascorbate-Mediated Enhancement of Reactive Oxygen Species Generation from 
Polymorphonuclear Leukocytes: Modulatory Effect of Nitric Oxide." Journal of Leukocyte Biology 75, no. 6 (June 2004): 1070-78. https://doi.org/10.1189/jlb.0903415.

3 42. Shi, Xin, Matthew D Sims, Michel M Hanna, Ming Xie, Peter G Gulick, Yong-Hui Zheng, Marc D Basson, and Ping Zhang. "Neutropenia during HIV Infection: Adverse Consequences and Remedies." International Reviews of Immunology 33, no. 6 (2014): 511-36. https://doi.org/10.3109/08830185.2014.893301.

43. Singh, Gurdeep, Andrew Brass, Christopher G. Knight, and Sheena M. Cruickshank. "Gut Eosinophils and Their Impact on the Mucus-Resident Microbiota." Immunology 158, no. 3 (2019): 194-205. https://doi.org/10.1111/imm.13110.

44. Sivanandham, Ranjit, Egidio Brocca-Cofano, Noah Krampe, Elizabeth Falwell, Sindhuja Murali Kilapandal Venkatraman, Ruy M. Ribeiro, Cristian Apetrei, and Ivona Pandrea.

15 45. Somsouk, Ma, Jacob D. Estes, Claire Deleage, Richard M. Dunham, Rebecca Albright, John 16 M. Inadomi, Jeffrey N. Martin, Steven G. Deeks, Joseph M. McCune, and Peter W. Hunt. "Gut 17 Epithelial Barrier and Systemic Inflammation during Chronic HIV Infection." AIDS (London, England) 29 , no. $1 \quad$ (January 2, 2015): 43-51. https://doi.org/10.1097/QAD.0000000000000511.

21 Xavier Anglaret. "Incidence of Neutropenia in HIV-Infected African Adults Receiving Co22 Trimoxazole Prophylaxis: A 6-Year Cohort Study in Abidjan, Côte d'Ivoire." Transactions of 23 the Royal Society of Tropical Medicine and Hygiene 100, no. 8 (August 2006): 785-90. 24 https://doi.org/10.1016/j.trstmh.2005.11.008.

47. Vono, Maria, Ang Lin, Anna Norrby-Teglund, Richard A. Koup, Frank Liang, and Karin Loré. "Neutrophils Acquire the Capacity for Antigen Presentation to Memory CD4+ T Cells in Vitro 
and Ex Vivo." Blood 129, no. 14 (06 2017): 1991-2001. https://doi.org/10.1182/blood-2016-

3 48. Weisgrau, Kim L., Logan J. Vosler, Nicholas L. Pomplun, Jennifer M. Hayes, Heather A.

4 Simmons, Kristen R. Friedrichs, and Eva G. Rakasz. "Neutrophil Progenitor Populations of

5 Rhesus Macaques." Journal of Leukocyte Biology 105, no. 1 (2019): 113-21.

$6 \quad$ https://doi.org/10.1002/JLB.1TA1117-431RR.

7 49. Weller, P F, W L Marshall, D R Lucey, T H Rand, A M Dvorak, and R W Finberg. "Infection,

8 Apoptosis, and Killing of Mature Human Eosinophils by Human Immunodeficiency Virus-1."

9 American Journal of Respiratory Cell and Molecular Biology 13, no. 5 (November 1995): 610-

10 20. https://doi.org/10.1165/ajrcmb.13.5.7576698.

11 50. Zevin, Alexander S., Lyle McKinnon, Adam Burgener, and Nichole R. Klatt. "Microbial 12 Translocation and Microbiome Dysbiosis in HIV-Associated Immune Activation.

13 [Miscellaneous Article]." Current Opinion in HIV \& AIDS 11, no. 2 (March 2016): 182-90.

14 https://doi.org/10.1097/COH.0000000000000234. 


\section{FIGURE LEGENDS:}

2 FIG1: Flow cytometric analysis of whole blood granulocyte surface markers. Flow

3 cytometric panel determining granulocyte subpopulations (A) shown using whole blood

4 samples. Frequencies of $\mathrm{CD} 64, \mathrm{CD} 32, \mathrm{CD} 16$, and $\mathrm{FcR} \varepsilon$ receptors on neutrophils

5 (green), eosinophils (blue), basophils (yellow), and monocytes (red), in naïve rhesus

6 whole blood samples (B). Baseline frequencies of granulocyte subsets in rhesus whole

7 blood samples (C): neutrophils (red), eosinophils (blue), and basophils (green)

8 determined by flow cytometry.

9 FIG 2: Granulocyte phenotypes analyzed by ImageStream. ImageStream flow

10 cytometry gating strategy (A) used to determine populations of neutrophils, eosinophils,

11 and basophils in whole blood. Image representation of (B) neutrophils (top), eosinophils

12 (middle), and basophils (bottom) isolated from rhesus whole blood.

13 FIG 3: Nuclear morphology of granulocyte subsets analyzed by ImageStream.

14 ImageStream flow cytometry images generated from whole blood samples and two

15 representative evaluations are shown (A and $\mathrm{B})$. Internalized DAPI for visualizing

16 nuclear morphology is overlaid with surface markers indicating neutrophils (top),

17 eosinophils (middle), and basophils (bottom).

18 FIG 4: Granulocyte subsets in whole blood of naïve and lentivirus-infected rhesus

19 macaques. Granulocyte subset frequencies determined in peripheral blood by flow

20 cytometry. Chronic SHIV infection whole blood samples (red) are compared to naïve

21 (blue). ${ }^{* *} \mathrm{P}<0.01,{ }^{* * *} \mathrm{P}<0.0001$, Mann-Whitney test.

22 FIG 5: Granulocyte subsets in mucosal and lymphoid tissues of naïve and

23 lentivirus infected macaques. Frequencies (A) of neutrophils (top), eosinophils 
1 (middle), and basophils (bottom) found in naïve (blue) and chronic SHIV (red) infected

2 rhesus macaque tissues. Frequencies (B) of neutrophils (left) and eosinophils (right)

3 found in vaginal (top) and colorectal (bottom) biopsies from naïve (blue) and chronic

4 SHIV (red) infected rhesus macaques analyzed using flow cytometry. ${ }^{*} \mathrm{P}<0.05$, ${ }^{* *} \mathrm{P}<$

$50.01,{ }^{* * *} \mathrm{P}<0.001$, Mann-Whitney test.

6 


\section{A}
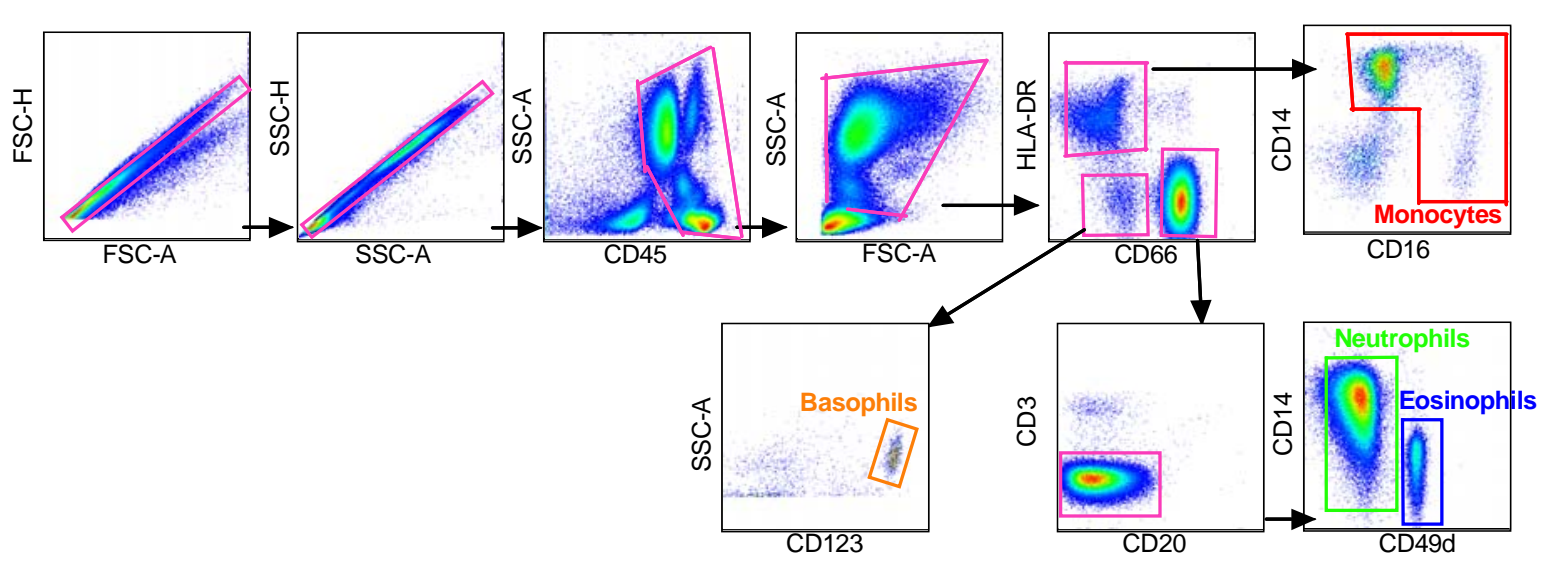

B

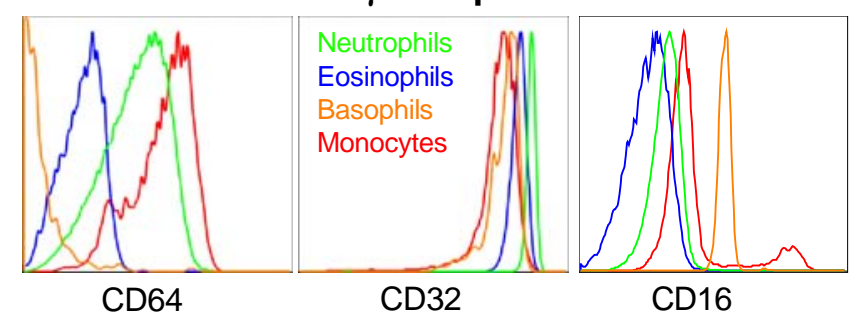

\section{Fc $\gamma$ Receptors}

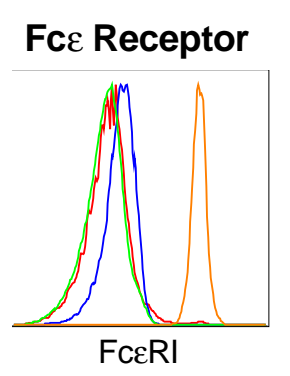

C

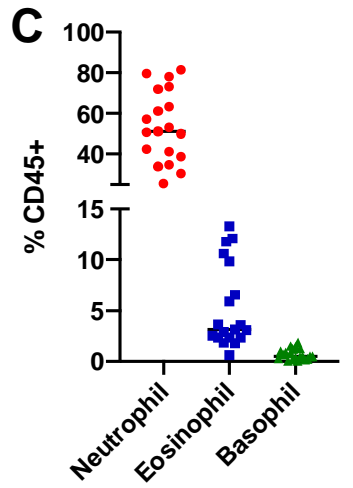




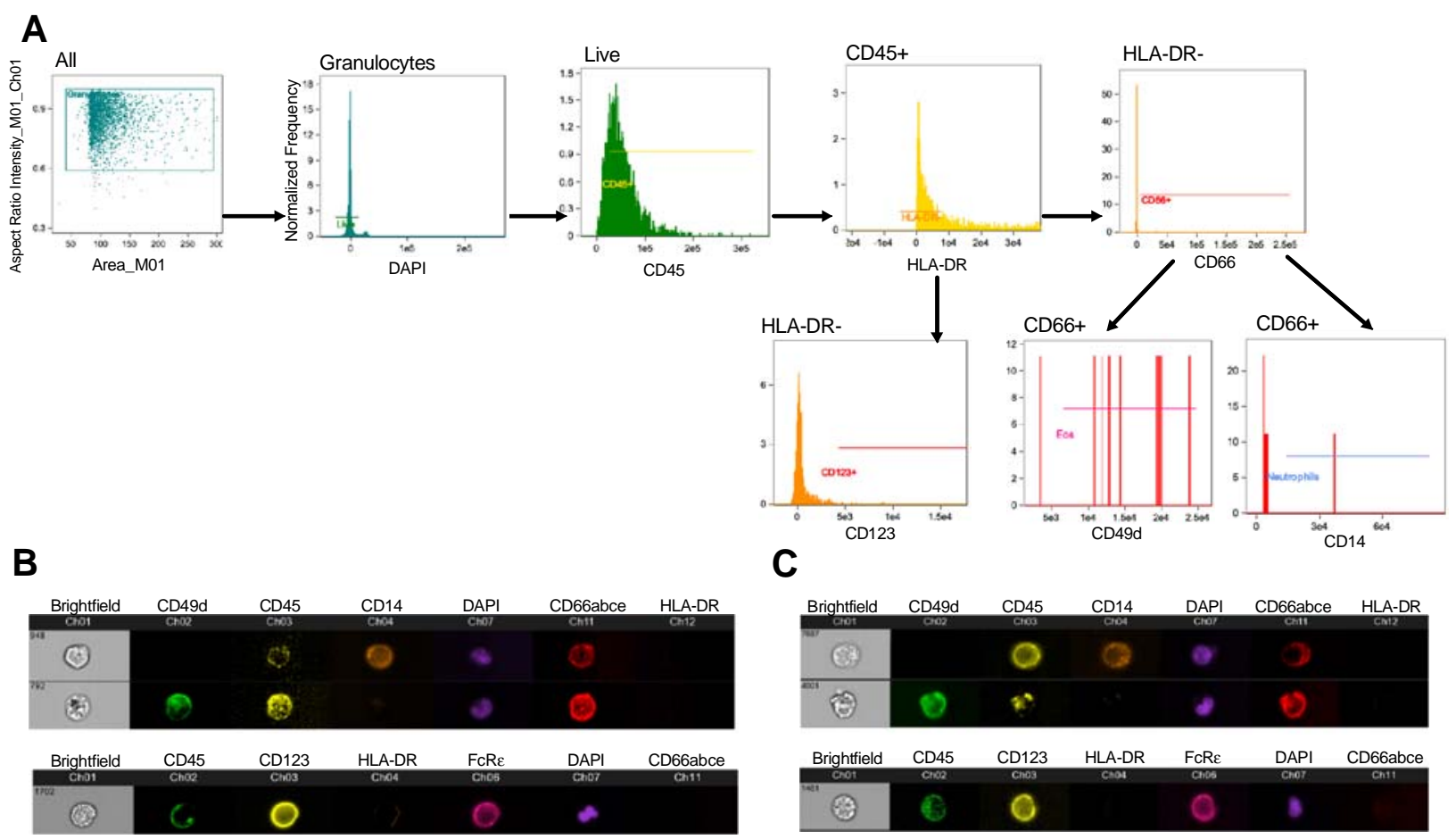




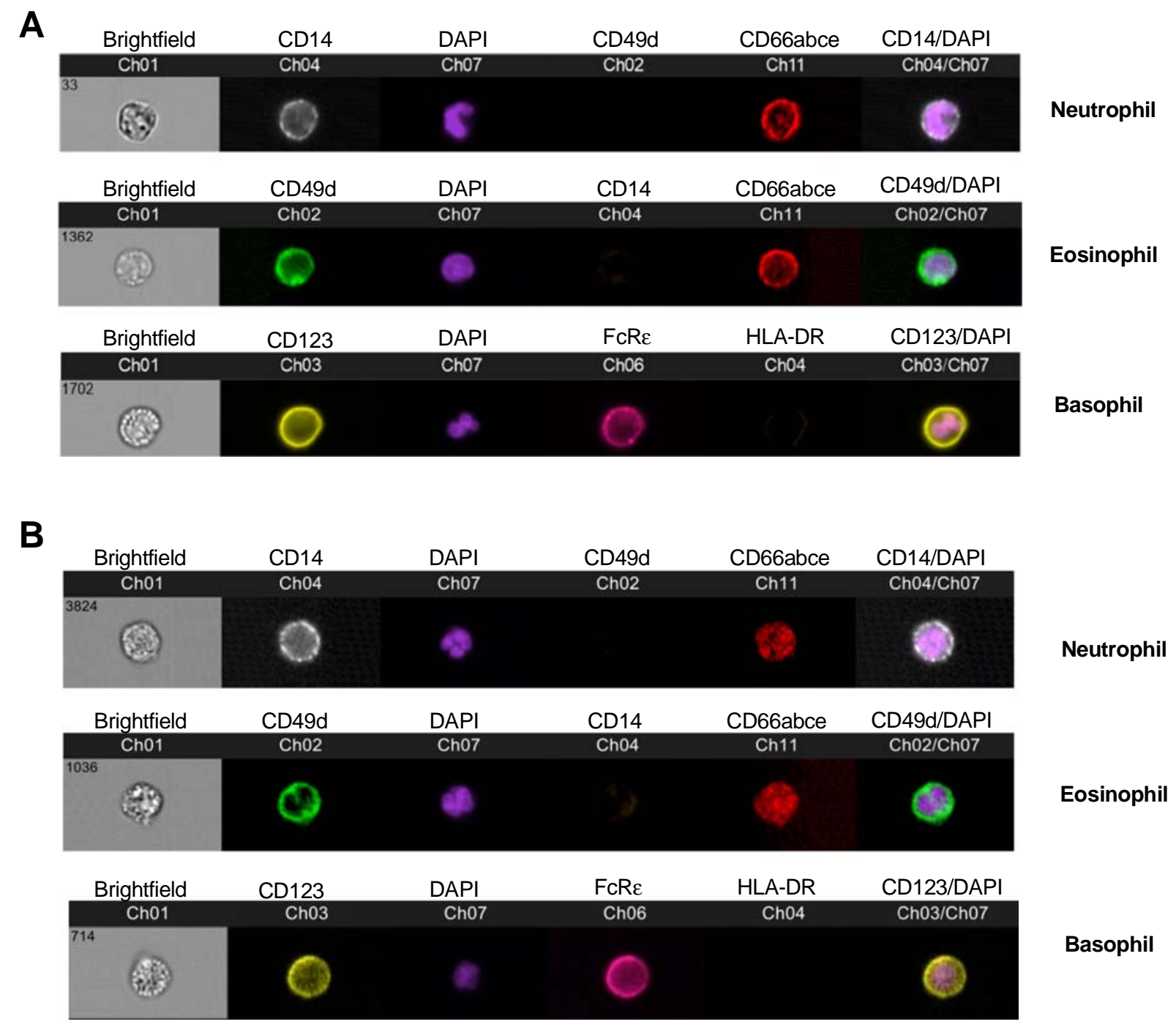




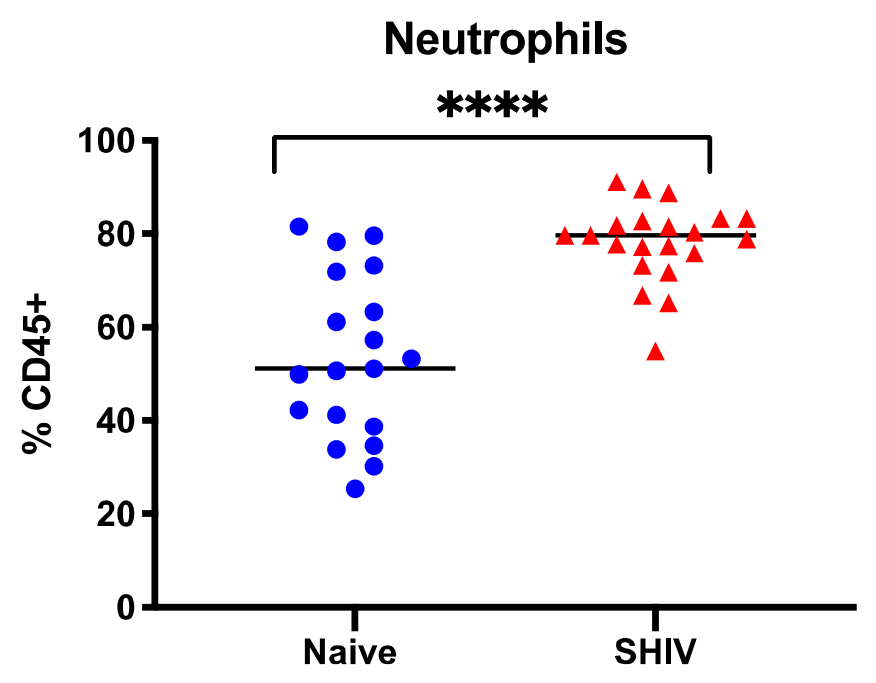

Eosinophils
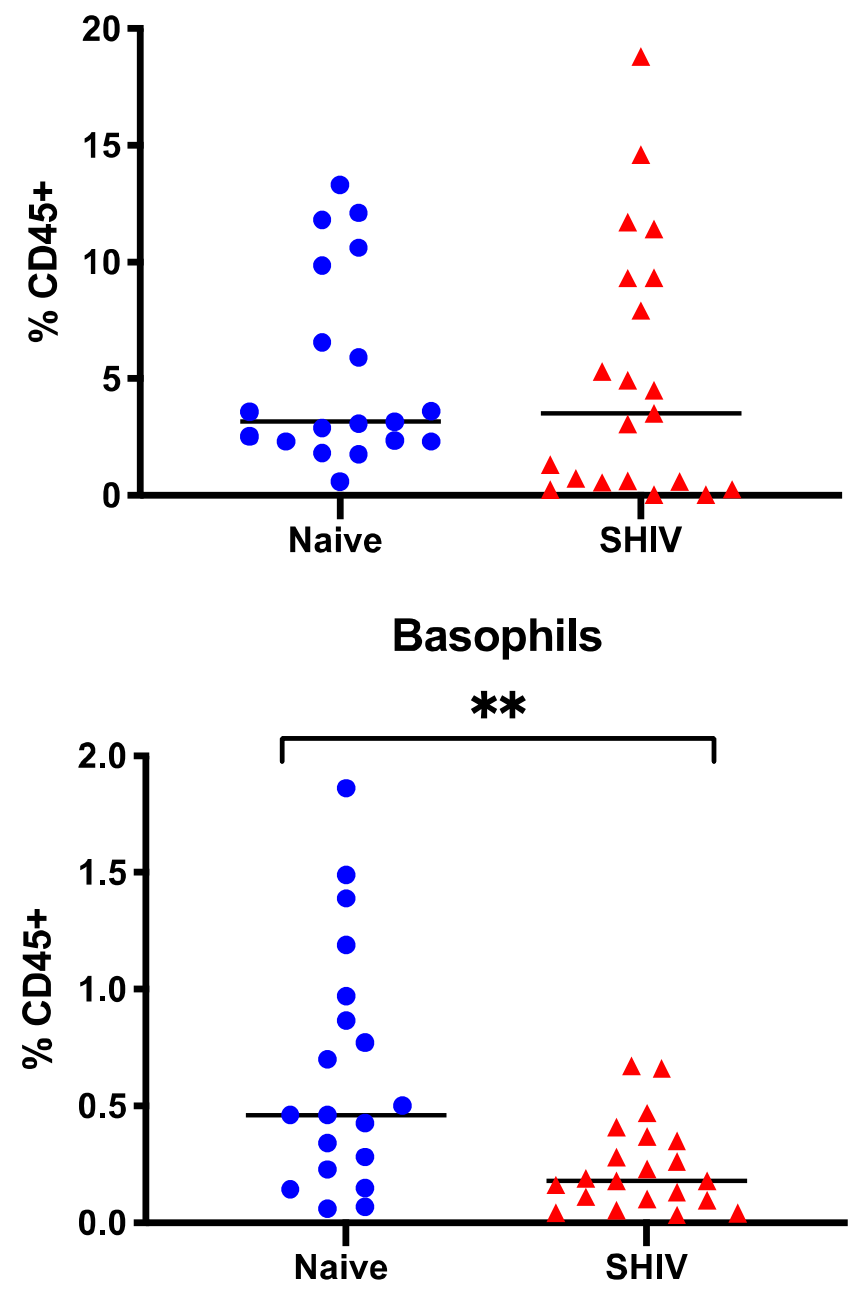


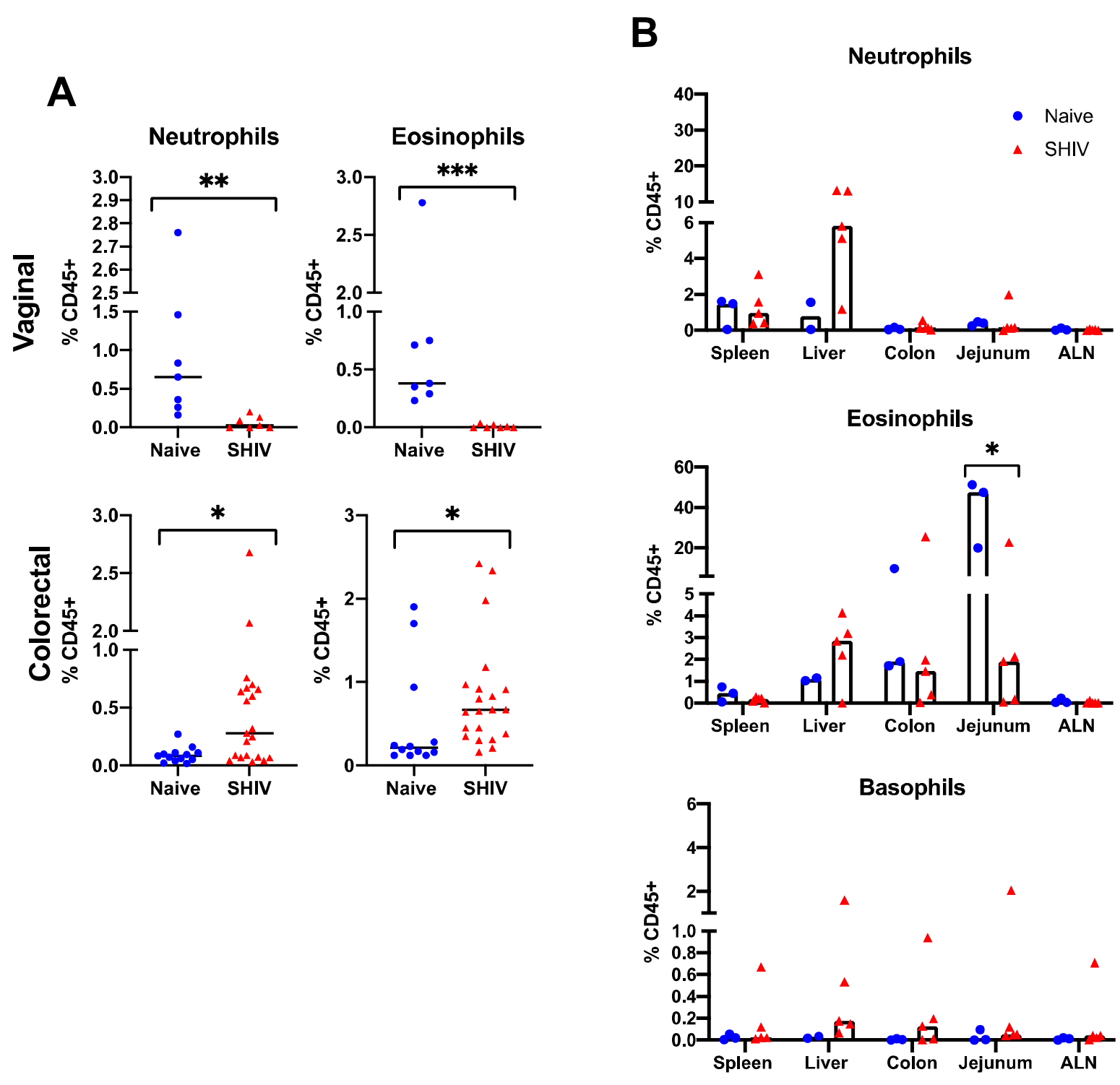

